\title{
The Two Facets of the Electromagnetic Interaction Term
}

\author{
Eliahu Comay \\ Charactell Ltd., Tel-Aviv, Israel \\ Email: elicomay@post.tau.ac.il
}

How to cite this paper: Comay, E. (2021) The Two Facets of the Electromagnetic Interaction Term. Open Access Library Journal, 8: e7617.

https://doi.org/10.4236/oalib.1107617

Received: June 7, 2021

Accepted: July 4, 2021

Published: July 7, 2021

Copyright () 2021 by author(s) and Open Access Library Inc.

This work is licensed under the Creative Commons Attribution International License (CC BY 4.0).

http://creativecommons.org/licenses/by/4.0/

\begin{abstract}
The significance of the interaction term of classical electrodynamics and quantum electrodynamics is analyzed. It is proven that this term is involved in the derivation of the equations of motion of the charge-carrying particles and the Maxwell equations of the electromagnetic fields. Classical electrodynamics, as well as quantum electrodynamics of Maxwell equations and the Dirac equation of a charged spin-1/2 particle, comply with the dual role of the interaction term. Inconsistencies arise from the equations of the Klein-Gordon particles, the electroweak theory of the $W^{ \pm}$, and the Proca theory of a massive photon. The experimental data and the incoherent structure of the mainstream literature substantiate these results.
\end{abstract}

\section{Subject Areas}

Particle Physics

\section{Keywords}

The Variational Principle, Electrodynamics, Equations of Motion, The Electroweak theory, The Klein-Gordon Theory, The Proca Lagrangian

\section{Introduction}

Physics aims to explain the processes of existing entities. It means that it assumes that the existence of these entities is independent of humans who create the science that explains the behavior of entities of nature. For example, physical theories explain that a copious amount of neutrinos are produced in a supernova explosion. And indeed, in the year 1987, neutrinos were measured in laboratories. These neutrinos are products of a supernova that exploded more than 160,000 years ago. It means that the supernova explosion is an event that is independent of its neutrino measurement on planet earth. It had taken place much 
before the beginning of civilization and before the experimenters have been born.

On the other hand, measurability is a fundamental principle of physics. It says that a genuine physical entity must produce an effect that can directly or indirectly be sensed by humans.

It means that a scientific theory must describe two different facets of a physical entity: its self-behavior and how it affects measurement devices that indicate the existence of that entity and its time evolution. A theory of a given particle achieves these objectives by two sets of expressions: one set describes a free particle and the other set accounts for its interaction with other physical entities.

This is not the full description of the problem. Indeed, physical processes abide by conservation laws. Assume that a measuring device signals an effect by means of an increase in its energy. Hence, the full system also comprises another physical entity whose energy decreases.

In principle, one may assume that the measured object directly affects the measuring device. In other cases, there is a mediating entity that connects between the measured object and the measuring device. An example of the first case is a photon interaction that excites atomic electrons or the Compton effect where a photon ionizes an atom. An electron-proton scattering is an example of the second case. Here electromagnetic fields mediate the interaction between these charged particles. Figure 1 illustrates the second case.

This work analyzes how these issues are embedded in the theoretical structure of electromagnetic systems. It examines classical electrodynamics that is derived from the variational principle [1] and Quantum Electrodynamics (QED) of electromagnetic fields and a charged Dirac particle. Theories of other kinds of particles are also tested.

The discovery of the charged pion illustrates the general argument presented herein. This discovery has used a photographic emulsion and the moving pion ionized atoms of this emulsion and produced a track of tiny bubbles (see [2], p. 4). A camera has recorded the track. Figure 2 illustrates this process.

Here are the actual elements of this experiment. The charged pion is the measured object. Its electromagnetic fields are the mediating entity (a quantum theory uses the electromagnetic 4-potential). Atoms of the photographic emulsion that interact electromagnetically with the fields of the charged pion are elements of the measuring device. The charged pion leaves a track of ionized atoms on which tiny bubbles appear.

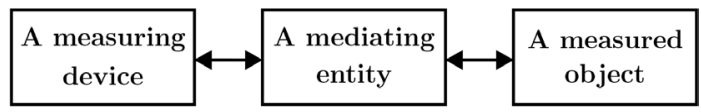

Figure 1. The three physical entities that are involved in measurement (see text).

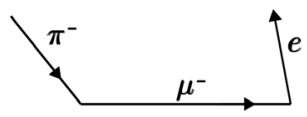

Figure 2. An illustration of the decay tracks of the $\pi^{-} \rightarrow \mu^{-} \rightarrow$ electron (see text). 
The main objective of this work is to analyze the theoretical structure of several electromagnetic systems and see if the corresponding theory describes this quite basic process. It is surprising to realize that too many textbooks dedicate a discussion to theories that do not abide by all elements of the physical principles that are pointed out above. This state of affairs indicates the timely need for this work.

Units where $\hbar=c=1$ are used. Greek indices run from 0 to 3. Most formulas take the standard form, and relativistic covariant expressions are generally used. The metric is diagonal and its entries are $(1,-1,-1,-1)$. The second section discusses classical electrodynamics that is base on the variational principle, and QED of Maxwellian electromagnetic fields and the Dirac electron. The third section examines other electromagnetic theories. The fourth section elaborates on the results of the previous sections. The Last section summarizes this work.

\section{The Theoretical Structure of Electrodynamics}

This section shows how classical electrodynamics and QED describe charged particles and electromagnetic fields in a form that fits the requirements that are presented in the introduction.

\subsection{The Classical Theory}

Landau and Lifshitz prove that an elementary classical particle is pointlike (see [1], pp. 46-47). Hence, classical electrodynamics takes a hybrid form: a Lagrangian describes the motion of an elementary pointlike charged particle and a Lagrangian density describes the time-dependence of electromagnetic fields. The action $\mathcal{S}$ is the appropriate integral of the charged particles' Lagrangian and the electromagnetic fields Lagrangian density (see [1], p. 73)

$$
\mathcal{S}_{C E D}=-\sum_{i} \int m_{i} \mathrm{~d} s-\sum_{i} \int e_{i} A_{\mu} \mathrm{d} x^{\mu}-\frac{1}{16 \pi} \int F_{\mu \nu} F^{\mu v} \mathrm{~d}^{4} x .
$$

Here the subscript $C E D$ denotes that the action $\mathcal{S}$ is that of Classical Electrodynamics, $s$ is the invariant time, the index $i$ runs on the massive pointlike particles, $m_{i}$ and $e_{i}$ are their mass and charge, respectively, $A_{\mu}$ is the electromagnetic 4-potential, and $F^{\mu v}$ denotes the tensor of the electromagnetic fields. The second term on the right hand side of (1) is the interaction term.

An appropriate variation of the action (1) yields the equations of motion of the charged particles (the Lorentz force) and the equations of motion of the electromagnetic fields (Maxwell equations). Thus, a variation of the particle's coordinates yields the Lorentz force

$$
m \frac{\mathrm{d} v^{\mu}}{\mathrm{d} s}=e F^{\mu v} v_{v}
$$

(see [1], pp. 49-51, 89; [3], p. 577). This is the equation of motion of a classical charged particle.

Furthermore, the 4-potential is the generalized coordinates of the electromagnetic fields (see [1], p. 78; [3], p. 595). The electromagnetic fields $F^{\mu v}$ is its de- 
rivatives in the form of a 4 -curl

$$
F_{\mu \nu}=A_{, \mu}-A_{\mu, v}
$$

(see [1], p. 65; [4], p. 33). In the case of fields, the general form of the Lagrangian density of a field function is

$$
\mathcal{L}\left(\psi(x), \psi(x)_{, \mu}\right)
$$

(see [5], p. 300). Here $x \equiv(t, \boldsymbol{x})$ denotes the four space-time coordinates. This expression is extended for cases of several kinds of field functions. The general form of the Euler-Lagrange equation is

$$
\frac{\partial \mathcal{L}}{\partial \psi_{r}}-\frac{\partial}{\partial x^{\mu}} \frac{\partial \mathcal{L}}{\partial\left(\partial \psi_{r} / \partial x^{\mu}\right)}=0
$$

where $\psi_{r}$ denotes the rth generalized coordinate (see [4], p. 16; [5], p. 300).

An application of (5) to the electromagnetic coordinates $A_{\mu}$ of (1) produces the inhomogeneous Maxwell equations

$$
F_{, v}^{\mu v}=-4 \pi j^{\mu}
$$

(see [1], p. 79; [3], p. 597; [4], p. 16). Here the last term of (1) yields the left hand side of (6) and the interaction term of (1) yields the right hand side of (6).

Conclusion A: The interaction term of the action of classical electrodynamics produces two equations of motion-the Lorentz force (2) that applies to a charged particle and the inhomogeneous Maxwell Equation (6) of the electromagnetic fields.

Implications of this result are the main topic of this work.

\subsection{Quantum Electrodynamics}

Let us examine how the variational principle is utilized in QED. The general community agrees that the Lagrangian density of an elementary quantum particle takes the form of (4). For example, a well-known textbook states that "all field theories used in current theories of elementary particles have Lagrangians of this form" (see [5], p. 300).

The four parameters of the quantum function $\psi(t, x)$ of (4) indicate that it can describe the probability of finding the particle at the space-time point $(t, x)$, but it cannot show how it is distributed around this space-time point. Hence, as stated above, like its classical counterpart, $\psi(t, \boldsymbol{x})$ describes an elementary pointlike particle.

As stated above, in the general case, the Lagrangian density depends on several kinds of quantum functions that describe the time-evolution of the particles of a given system. For example, the Lagrangian density of QED is (see e.g. [4], p. 78; [6], p. 84)

$$
\mathcal{L}_{\mathrm{QED}}=\bar{\psi}\left[\gamma^{\mu} i \partial_{\mu}-m\right] \psi-e \bar{\psi} \gamma^{\mu} \psi A_{\mu}-\frac{1}{16 \pi} F_{\mu \nu} F^{\mu \nu},
$$

where $\bar{\psi}=\psi^{\dagger} \gamma^{0}$ are the Dirac functions. Like in the classical case (1), the QED 
Lagrangian density (7) uses just one interaction term. It is the term of (7) that contains $A_{\mu}$. Moreover, the entire QED Lagrangian density (7) is analogous to the classical expression (1). Here quantities of the classical point particle are replaced by expressions that depend on the Dirac function $\psi$. In particular, the 4-current of a Dirac particle

$$
j^{\mu}=\bar{\psi} \gamma^{\mu} \psi
$$

is conserved

$$
j_{, \mu}^{\mu}=0
$$

(see e.g. [4], pp. 50, 51; [5], p. 10).

A variation of the QED Lagrangian density (7) with respect to $\bar{\psi}$ yields the Dirac equation

$$
\left(i \gamma^{\mu} \partial_{\mu}-e \gamma^{\mu} A_{\mu}-m\right) \psi=0
$$

(see [4], p. 78; [6], p. 85). The same equation is obtained from a variation of $\psi$.

The electromagnetic quantities $A_{\mu}$ and $F^{\mu \mu}$ of (1) of the classical case are analogous to those of (7) of QED. They take the same formal form: a term that contains a quadratic quantity of $F^{\mu v}$ and another term that is proportional to $A_{\mu}$. Hence, the mathematical derivation of the electromagnetic fields equations is analogous to the classical case and the 4-current of the Dirac electron (8) yields the quantum version of the inhomogeneous Maxwell equations

$$
F_{, v}^{\mu v}=4 \pi e \bar{\psi} \gamma^{\mu} \psi
$$

It is important to note that QFT textbooks show both the Dirac equation of a charged spin-1/2 particle (10) and the Maxwell equations of the electromagnetic fields (11) (see [4], p. 78; [6], p. 85).

This analysis proves that Conclusion A of the classical case also holds for QED: one interaction term yields the equation of motion of the massive charged particle and Maxwell equations of the electromagnetic fields.

Here is a point that is required for later analysis. The first term of the Euler-Lagrange Equation (5) indicates that its dependence on a generalized coordinate takes the form of a derivative with respect to this coordinate. This is the basis of the following conclusion.

Conclusion B: If $A$ is a generalized coordinate of a Lagrangian density that contains a term whose form is $G A^{n}$ and $n>1$ then the corresponding Euler-Lagrange equations explicitly depend on this generalized coordinate.

As stated above, the 4-potential is regarded as the generalized coordinate of the electromagnetic fields. The classical action (1) and the QED Lagrangian density (7) contain no term whose form is $G A^{n}$ and $n>1$. Therefore, the classical Maxwell Equations (6) and their quantum version (11), are free of the electromagnetic 4-potential $A_{\mu}$.

By definition, an electromagnetic theory is gauge invariant if it is invariant under a transformation of the 4-potential 


$$
A_{\mu} \rightarrow A_{\mu}+\alpha(x)_{, \mu},
$$

where $\alpha(x)$ is an arbitrary function of the space-time coordinates (see [5], p. 342 ). It follows that the electromagnetic fields (3) are gauge invariant. Furthermore, the classical version of Maxwell equations (6), as well as their quantum version (11), are independent of the 4-potential $A_{\mu}$. A fortiori, they are independent of any variation of $A_{\mu}$. The independence of Maxwell equations on $A_{\mu}$ proves this important result:

Conclusion GI: Maxwell equations of the electromagnetic fields are gauge invariant because they are independent of the 4 -potential $A_{\mu}$.

\section{Other Theories}

Mainstream QFT textbooks discuss other theories of elementary charged particles. The Lagrangian density of a set of these theories contains a typical quadratic mass term of the form

$$
\mathcal{L}=m^{2} \phi^{2}+\cdots
$$

Hereafter, a theory that belongs to this set is called a second order theory.

Dimension is an intrinsic attribute of a physical quantity. In the units where $\hbar=c=1$, the action is dimensionless and the dimension of the Lagrangian density is $\left[L^{-4}\right]$. It follows that the dimension of the Dirac function $\psi$ of (7) is $\left[L^{-3 / 2}\right]$. On the other hand, the dimension of the field function $\phi$ of a second order theory (13) is $\left[L^{-1}\right]$. It means that the mathematical structure of a quantum function of a second order theory intrinsically differs from the Dirac electronic function of the QED theory that is described in subsection 2.2.

The expression for the electromagnetic interaction of a second order theory of an elementary charged quantum particle has a term that can be briefly written in this form

$$
\mathcal{L}_{\text {int }}=a \phi^{\dagger} \chi A A
$$

Here $a$ is a numerical coefficient, $\phi^{\dagger}$ and $\chi$ denote components of the quantum function of a charged particle, and $A$ is the electromagnetic 4-potential. The indices that show how this expression is contracted and produce a Lorentz scalar are removed. In the case of the KG theory $\phi^{\dagger}, \chi$ are the KG field function $\phi$ (see [7], p. 198; [8], p. 73). In the case of the electroweak theory of the $W^{ \pm}$particle, $\phi^{\dagger}, \chi$ are components of the $W^{ \pm} 4$-vectors (see [8], p. 113; [9], p. 518). Here the product of the 4-potential $A A$ plays the primary role. Hence, the Proca theory of a massive photon can also be included in the discussion of this set of theories, because its Lagrangian density contains a mass term of the form $a m^{2} A_{\mu} A^{\mu} \quad$ (see [3], p. 598).

Let us examine the electromagnetic fields equations of a second order theory of an elementary charged quantum particle that is discussed in this section. These equations should be consistent with Maxwell equations because there is a unique kind of electric charge in Nature and it is embedded in Maxwellian elec- 
trodynamics. As pointed out after (6), the pure electromagnetic field term $-\frac{1}{16 \pi} F_{\mu \nu} F^{\mu \nu}$ yields the left hand side of Maxwell Equation (6). However, in the present case, the quadratic quantity $A A$ of (14) yields fields equations of this form

$$
F_{, v}^{\mu v}=G A+\cdots,
$$

where $G$ depends on the quantum functions of the massive charged particle. The explicit dependence of the right hand side of (15) on the electromagnetic 4-potential $A_{\mu}$ proves that it is a gross violation of Maxwellian electrodynamics.

Conclusion C: The Lagrangian density of a second order theory of an electrically charged particle contains a term that has the product $A A$ of the 4-potential. Results of this term violate Maxwellian electrodynamics because these equations are independent of the 4-potential $A_{\mu}$. This conclusion applies to the KG equation, the electroweak $W^{ \pm}$theory, and also to the Proca theory of a massive photon.

\section{Discussion}

Maxwell equations have impressive experimental support. For example, in Maxwellian electrodynamics the photon is massless. The experimental upper bound on the photon's mass is smaller than $10^{-18} \mathrm{ev}$ [10]. Namely, the experimental upper bound on the photon's mass is smaller than $10^{-23}$ times the electronic mass. Hence, experiment supports this attribute of Maxwellian electrodynamics and denies the Proca idea of a massive photon.

Maxwell equations distinguish between two different electromagnetic objects: massless electromagnetic fields and massive charge-carrying particles. Here electromagnetic fields do not carry a charge. This attribute has an extremely strong experimental support, where the upper bound on the photon's charge is $q<10^{-35}|e|[10]$.

Moreover, the Coulomb law is embedded in Maxwellian electrodynamics (see [1], pp. 95, 96; [3], pp. 217-218). Here a test of the Coulomb law examines $\varepsilon$ of this expression for the electric field of a motionless charge

$$
E=Q / r^{(2+\varepsilon)} .
$$

The data prove that the experimental upper bound on $|\varepsilon|$ is extremely negligible: $|\varepsilon|<10^{-16}$ (see [11], p. S143). This is another strong experimental support for Maxwellian electrodynamics.

The Coulomb potential $e / r$ of (16) plays a crucial role in the experimental confirmation of QED. Indeed, the Dirac theory of the quantum states of the hydrogen atom uses this potential, and the data fit calculations of this theory for more than 5 decimal digits (see [12], section 4.4 on p. 52).

Conclusion D: The data support Maxwellian electrodynamics. This evidence denies the KG equation, the electroweak $W^{ \pm}$theory, and the Proca 
theory of a massive photon, because their equations for the electromagnetic fields explicitly depend on the 4-potential $A_{\mu}$

Conclusion E: This outcome means that the electromagnetic fields equations of second order theories violate gauge invariance. This result is inconsistent with the conclusion GI of Maxwell equation (see at the end of section 2).

It is interesting to note that an analogous assertion concerning the violation of gauge invariance can be found in a well-known mainstream QFT textbook (see [5], p. 343).

An observation of QFT textbooks shows that they do not treat second order theories of a charged particle like their treatment of the Dirac theory of a spin-1/2 charged particle. The majority (if not all) of QFT textbooks that discuss the Dirac theory show a coherent expression for the electromagnetic interaction of a charged Dirac particle and electromagnetic fields. Furthermore, they show the explicit form of the partial differential equations of the physical entities: the Maxwell equations of the electromagnetic fields and the Dirac equation of a charged spin-1/2 particle. In contrast, only a few QFT articles and textbooks show explicit expressions for the electromagnetic interaction of a charged KG particle ([4], p. 312; [7], p. 198; [8], p. 73; [12], p. 189; [13], p. 140). However, none of these textbooks presents a comprehensive analysis of the problem and derives the electromagnetic fields equations. This quite unusual situation indicates the problematic structure of the KG theory of an elementary charged particle. The inconsistency of its theoretical structure that is proved above explains this rather strange state of affairs, where a theory of a charged particle ignores the full structure of its electromagnetic interaction. This work fills this gap and proves that the interaction terms of these theories are inherently wrong.

Remark: it is explained in the second paragraph of subsection 2.2 that a quantum function of the form $\psi(t, \boldsymbol{x})$ describes an elementary pointlike particle. Hence, the pions are not KG particles, because they comprise a $\bar{q} q$ pair of quarks of the $u, d$ flavor. Furthermore, experiments prove that pions are not pointlike, and the charge radius of a $\pi^{+}$is not much smaller than that of the proton [10]. It is interesting to note that the official particle report [10] shows no elementary charged particle that satisfies the KG equation. It means that with respect to the denial of the KG theory of a charged particle, the theoretical analysis that is presented above goes together with relevant experiments.

The KG and the electroweak theory of the $W^{ \pm}$share the troublesome expression (14). This is the reason that explains why the literature indicates analogous problematic issues of the electromagnetic interactions of the electroweak $W^{ \pm}$. For example, the Dirac equation (or its nonrelativistic limit-the Schroedinger equation) is successfully used for every spin-1/2 charged quantum particle. In contrast, most QFT textbooks refrain from a discussion of the electromagnetic interaction of the electroweak $W^{ \pm}$. This is the reason for the situation where more than 40 years after establishing the electroweak theory, large research centers like Fermilab and CERN still use a phenomenological expression for the $W^{ \pm}$ 
electromagnetic interaction [14] [15]. The theoretical inconsistency of the electromagnetic interactions of the electroweak $W^{ \pm}$is proved in this work. This proof explains this quite strange discrepancy of the mainstream QFT incomplete treatment of the electromagnetic interaction of the electroweak $W^{ \pm}$theory.

On top of these issues, two other contradictions follow the troublesome expression (14):

1) As stated above, Maxwell equations prove that electromagnetic fields do not carry a charge. Moreover, a quantum expression of a charge-carrying object that is used in a Lagrangian density should have the dimension $\left[L^{-3}\right]$ of charge density. In contrast, the troublesome expression (14) contains a product of two quantum functions of a second order theory, and the dimension of this product is $\left[L^{-2}\right]$. This unacceptable inconsistency of dimension disproves the KG theory and the electroweak theory of the $W^{ \pm}$.

2) The right hand side of the inhomogeneous Maxwell equations (6) and (11) is proportional to the electric charge $e$. By contrast, the troublesome expression (14) contains the quadratic factor $e^{2}$ ([7], p. 198; [8], p. 73, 113; [9], p. 518; [12], p. 189; [13], p. 140). This is a sheer violation of Maxwellian electrodynamics.

\section{Concluding Remarks}

This work points out the significance of the variational principle as the cornerstone of the theoretical structure of classical electrodynamics and the standard form of QED. Properties of the charge-fields interaction term are analyzed. It is proven that this term contributes to the structure of the equation of motion of the charged particle and Maxwell equations of the electromagnetic fields. This outcome is consistent with experiments and provides the theoretical basis for the amazing technological achievements of our time.

The case of the Lagrangian density of a second order theory is different. Here contradictory results are obtained from the theory of a charged KG particle, the electroweak theory of the $W^{ \pm}$particle, and the Proca theory of a massive photon. A peculiar result of the electromagnetic field equations of these theories violates gauge invariance. It turns out that SM textbooks do not show a coherent treatment of the problem. For example, some of them present inconsistent expressions for the electromagnetic interaction (see e.g. [8], p. 113; [9], p. 518). On the other hand, other SM textbooks simply ignore this topic. Furthermore, unlike the case of QED, none of them shows a comprehensive discussion of the electromagnetic field equations. This state of affairs proves the problematic status of theories of a charged KG particle, the electroweak theory of the $W^{ \pm}$particles, and the Proca theory of a massive photon. Conclusions D, E are written few lines below Equation (16) briefly describe these matters.

It is interesting to note that the results of this work comply with Dirac's lifelong objection to second-order quantum equations [16].

\section{Conflicts of Interest}

The author declares no conflicts of interest. 


\section{References}

[1] Landau, L.D. and Lifshitz, E.M. (2005) The Classical Theory of Fields. Elsevier, Amsterdam.

[2] Perkins, D.H. (1987) Introduction to High Energy Physics. Addison-Wesley, Boston.

[3] Jackson, J.D. (1975) Classical Electrodynamics. John Wiley, New York.

[4] Peskin, M.E. and Schroeder, D.V. (1995) An Introduction to Quantum Field Theory. Addison-Wesley, Boston.

[5] Weinberg, S. (1995) The Quantum Theory of Fields, Vol. I. Cambridge University Press, Cambridge.

[6] Bjorken, J.D. and Drell, S.D. (1965) Relativistic Quantum Fields. McGraw-Hill, New York. https://doi.org/10.1063/1.3047288

[7] Pauli, W. and Weisskopf, V. (1994) Helvetica Physica Acta, 7, 709 (1934). English translation: Miller, A.I. Early Quantum Electrodynamics. Cambridge, University Press, 188-205. https://doi.org/10.1017/CBO9780511608223.017

[8] Cottingham, W.N. and Greenwood, D.A. (2007) An Introduction to the Standard Model of Particle Physics. Second Edition, Cambridge University Press, Cambridge.

[9] Sterman, G. (1993) An Introduction to Quantum Field Theory. Cambridge University Press, Cambridge. https://doi.org/10.1017/CBO9780511622618

[10] Zyla, P.A., et al. (2020) Particle Data Group. Progress of Theoretical and Experimental Physics, 83, C01.

[11] Tu, L.C. and Luo, J. (2004) Experimental Tests of Coulomb's Law and the Photon rest Mass. Metrologia, 41, S136.

https://www.researchgate.net/publication/228689980 https://doi.org/10.1088/0026-1394/41/5/S04

[12] Bjorken, J.D. and Drell, S.D. (1964) Relativistic Quantum Mechanics. McGraw-Hill, New York.

[13] Schwartz, M.D. (2020) Quantum Field Theory and the Standard Model. Cambridge University Press, Cambridge.

[14] Abazov, V.M., et al. (2012) D0 Collaboration. Physics Letters, B718, 451.

[15] Aad, G., et al. (2012) ATLAS Collaboration. Physics Letters, B712, 289.

[16] Dirac, P.A.M. (1978) Mathematical Foundations of Quantum Theory. Academic, New York. https://doi.org/10.1016/B978-0-12-473250-6.50005-4 